\title{
Limit loads for a shallow anchor/trapdoor embedded in a non-associative Coulomb soil
}

\author{
C. C. SMITH*
}

\begin{abstract}
A theoretical methodology for determining the range of possible plane-strain limit loads acting on an anchor or trapdoor buried within a non-associative Coulomb soil is presented. Solutions are given for shallow anchors and trapdoors underlying both weightless soil with surface load and soil possessing self-weight with no surface load. A method for determining limit loads for combined scenarios is additionally outlined. Solutions are shown to correlate favourably with experimental and finite-element data in the literature.
\end{abstract}

KEYWORDS: anchors; limit state design/analysis; plasticity
Cette communication présente une méthodologie théorique pour la détermination de la plage de charges limites de déformation plane agissant sur un dispositif d'ancrage ou une trappe enterrés dans un sol de Coulomb non associatif. Des solutions sont proposées pour des dispositifs d'ancrage ou des trappes à faible profondeur placés sous un sol dépourvu de poids avec charge surfacique, et un sol possédant son propre poids sans charge surfacique. En outre, on présente également une méthode de détermination des charges limites pour des scénarios mixtes. Les solutions présentent une bonne corrélation avec des données expérimentales et aux éléments finis contenues dans des documents techniques sur ce sujet.

\section{INTRODUCTION}

An associative geotechnical limit analysis requires that the angle of dilation $\psi$ of a soil be taken equal to the angle of shearing resistance $\phi$. This assumption underpins the powerful upper- and lower-bound theorems of plasticity, and the existence of a unique limit load. However, it is known that real soils are non-associative with dilation angle $\psi<\phi$. If a soil is modelled as a non-associative plastic material, then it is found that: (a) the limit load is not unique, and may be dependent on initial conditions and stress path to failure; (b) the limit load will always be less than or equal to the associative limit load (Chen, 1975).

Non-associativity can potentially reduce the limit load through two main processes: (a) a direct change to the collapse kinematics; (b) softening in shear bands (due purely to non-associativity), as discussed for example by Vermeer (1990).

These observations apply to materials that are rigid and perfectly plastic, with a non-associated flow rule, and which do not require the additional consideration of (true) strainsoftening (e.g. transition from peak to critical state strength), which is beyond the scope of this paper.

\section{Static determinacy}

Although there exist a large number of associative solutions in the literature to a wide range of geotechnical problems, very few closed-form solutions exist for nonassociative limit loads. Drescher \& Detournay (1993), for example, considered the kinematic analysis of statically determinate non-associative problems on the assumption that full (non-associative) strain-softening was able to manifest itself along slip planes. Without strain-softening, the collapse load for a statically determinate mechanism may otherwise be unaffected by non-associativity.

This paper, in contrast, examines the class of statically

Manuscript received 5 December 2010; revised manuscript accepted 22 September 2011. Published online ahead of print 19 May 2012.

Discussion on this paper closes on 1 December 2012, for further details see p. ii.

* Geotechnical Engineering Group, Department of Civil and Structural Engineering, University of Sheffield, UK. indeterminate problems, and specifically the anchor/trapdoor problem. In these cases, static indeterminacy provides one vehicle by which the non-associative and associative solutions can differ. This indeterminacy manifests itself in the anchor/trapdoor problem, in that the horizontal stresses are not constrained to a single value at collapse. Such problems are attractive for the derivation of a closed-form solution, in that they retain a useful degree of simplicity.

The primary aim of this paper is to illustrate how the range of possible non-associative limit loads might be bracketed in the context of closed-form solutions to the anchor/trapdoor problem.

\section{DERIVATION OF A NON-ASSOCIATIVE SOLUTION}

The procedure outlined by Davis (1968) is adopted. An admissible plastic stress distribution is first generated, to which a corresponding pattern of velocity characteristics is mapped according to the appropriate flow rule. A distribution of velocities corresponding to this pattern of velocity characteristics and satisfying the deformation boundary conditions is then derived. For the solution to be considered admissible (although not necessarily unique), the stress distribution must extend over the whole soil mass in a statically admissible manner without exceeding the strength. Additionally, as pointed out by Drescher \& Detournay (1993), it is necessary to confirm that the combined stress and velocity fields are thermodynamically admissible.

In a previous paper, Smith (1998) presented a rigorous, almost identical set of upper- and lower-bound solutions applicable to the anchor/trapdoor problem in an associative Coulomb material. These solutions will be used as the basis of the derivation of non-associative limit loads. Simple solutions involving only one velocity discontinuity at each edge of the trapdoor will be adopted.

The condition of co-axiality is adopted in this paper. In this case, the principal axes of strain increment and stress are assumed to be coincident. Solutions are derived for both weightless soil with surface load and soil possessing selfweight with no surface load. The former case lends itself more readily to straightforward analysis, and will be used to explore the solution space in detail, whereas the latter case, 
which is of more general practical relevance, will be examined in a single context.

A comparison is then made with finite-element and experimental data in the literature to contextualise the derived solutions. Finally, examination of translational failure mechanism analysis for the trapdoor problem is undertaken, which leads to a method for determining limit loads for combined surface load and self-weight scenarios.

\section{PROBLEM DEFINITION}

It is required to find compatible equilibrium stress and velocity fields consistent with the stress and displacement boundary conditions relevant to a rigid anchor/trapdoor of width $B$ underlying a uniform body of cohesionless soil of angle of shearing resistance $\phi$, unit weight $\gamma$ and depth $D$, as depicted in Fig. 1. The surface of the soil above the anchor/trapdoor carries a uniform vertical surcharge load $q \geqslant 0$.

The solutions presented in this paper will be restricted to the plane-strain case of a horizontal anchor/trapdoor. Following the conventions applied in a previous paper (Smith, 1998), the term 'trapdoor' will be used to indicate both an anchor (passive) and a trapdoor (active). Strictly, the solutions are directly applicable only to a trapdoor in a basement layer. The behaviour of an anchor plate will differ, in that a rising anchor plate leaves a void beneath itself, allowing localised flow around the anchor. This may affect the limit load, and will usually affect large-displacement loading, but is beyond the scope of this paper.

Solutions will be derived in terms of stress state described by mean stress $\sigma$ and major principal stress direction $\xi$ measured anticlockwise from the negative $x$-axis. In this framework the magnitude of the major principal stress is given by $\sigma(1+\sin \phi)$.

\section{WEIGHTLESS SOIL WITH A SURFACE LOAD \\ Solution characteristics}

Where the soil is assumed to have zero self-weight $(\gamma=0)$ and uniformly distributed properties of angle of shearing resistance $(\phi)$ and angle of dilation $(\psi)$, then the limit load $F$ per unit length for the anchor or trapdoor may be expressed in a dimensionless form as

$$
\frac{F}{q B}=1 \pm 2 \frac{D}{B} \Upsilon(\phi, \psi)
$$

where the sign depends on whether displacement is active $(-)$ or passive $(+)$.

The same general arguments as given by Smith (1998) for a soil possessing self-weight may be applied to this scenario, and are briefly summarised here. The attributes of a stress field compatible with an underlying displacing trapdoor within a rigid basement layer are as follows.

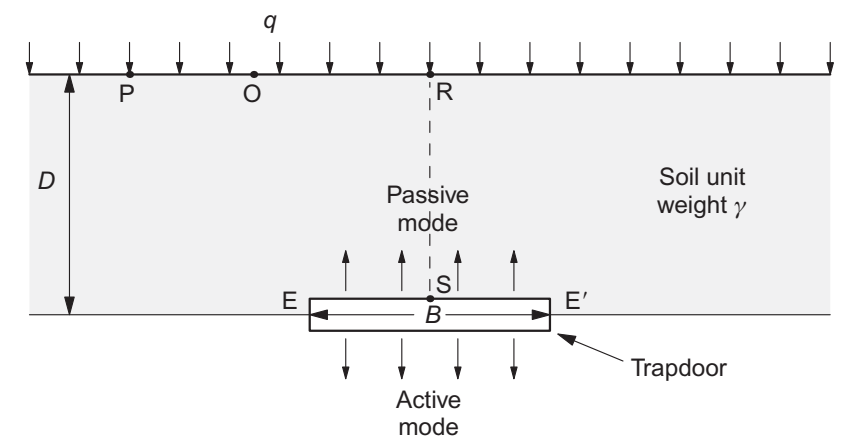

Fig. 1. The trapdoor problem: active and passive modes (a) The orientation of the principal stresses within the soil just beneath the soil surface can only be vertical or horizontal, and will generally differ for soil lying directly above the trapdoor (e.g. in the region of R in Fig. 1) and for soil lying some distance to the side (e.g. in the region of $\mathrm{P}$ ). A transition zone (in the region of $\mathrm{O}$ ) must therefore exist between the two.

(b) The direction of shear stresses along, for example, a line joining $\mathrm{O}$ and $\mathrm{E}$ in Fig. 1 must act as to oppose the soil motion (this ensures thermodynamic admissibility).

(c) Any velocity characteristics in the problem must pass through a zone in which the stress field is limiting.

For the purposes of the initial analysis, a shallow trapdoor will be considered, and is defined as one buried at a sufficiently small embedment ratio $(D / B)$ such that the soil stress fields generated at each edge ( $E$ and $\left.E^{\prime}\right)$ of the trapdoor do not interact. A complete analysis therefore requires the investigation of the stress field at one edge only, as shown in Fig. 2, and will be applicable to both active and passive trapdoors, depending on whether point $\mathrm{E}$ is taken as the left- or right-hand edge of the trapdoor.

The simplest plausible stress and velocity field for this problem compatible with the required attributes above is one that is scale independent or geometrically self-similar with vertical or horizontal major principal stresses at the surface and varying only with angle about $\mathrm{O}$, and with a single velocity discontinuity $\mathrm{OE}$ separating two rigid blocks, where $\mathrm{E}$ is the edge of the trapdoor. This stress field also requires the assumption of a partially rough basement layer with interface friction $\delta$ typically less than $\phi$.

Although an infinite number of continuous stress fields exist that satisfy such conditions, a much simpler, but still broadly representative, subset of solutions can be examined through the use of a small number of stress discontinuities.

As depicted in Fig. 2, an arrangement of three discontinuities $\mathrm{OA}_{1}, \mathrm{OA}_{2}$ and $\mathrm{OA}_{3}$ can be shown to give the required form of equilibrium stress field. (E may lie either side of $\mathrm{A}_{2}$.) This produces a considerably simpler solution than for the self-weight problem, as there are only four distinct stress states in zones $0,1,2$ and 3 , where zone $i$ represents the area $\mathrm{A}_{i} \mathrm{OA}_{i+1}$. Let discontinuity $\mathrm{A}_{i}$ be orientated at angle $\theta_{i}$ anticlockwise from $\mathrm{OA}_{0}$, and the mean stress and major principal stress direction (measured anticlockwise from $\mathrm{OA}_{0}$ ) in each zone $i$ be denoted $\sigma_{i}$ and $\xi_{i}$ respectively. From the problem definition, $\xi_{0}$ is thus equal to $90^{\circ}$ and $\xi_{3}=0^{\circ}$. Limiting conditions are required only in the zone $(j)$ in which the velocity characteristic (OE in Fig. 2) is manifested: thus each zone $i$ may be assigned a mobilised angle of shearing resistance $\phi_{i}^{\mathrm{m}} \leqslant \phi$, with the condition that $\phi_{j}^{\mathrm{m}}=\phi$. Derivation of the equations that may be used to

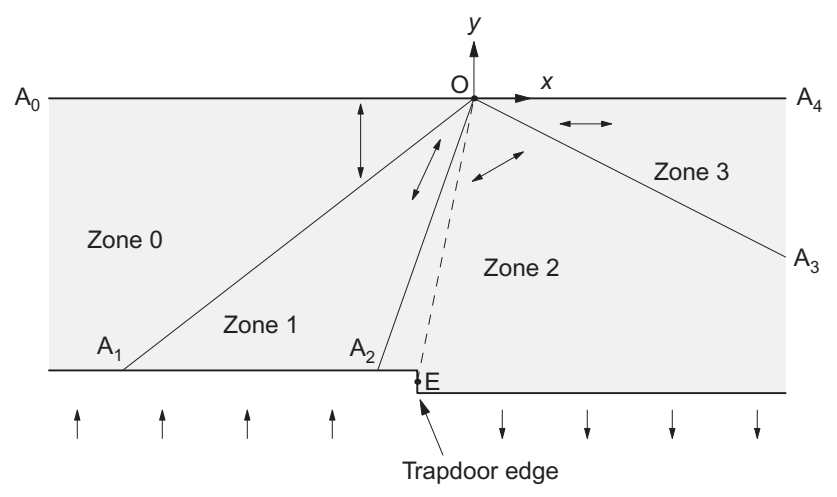

Fig. 2. General form of the stress fields at one edge of the trapdoor. Double-headed arrows indicate major principal stress directions 
relate the stress states in adjacent zones is given in Appendix 1.

Normally it would be expected that the velocity discontinuities would emerge at an angle $45 \pm \psi / 2$ to the surface. However, for the proposed solution, this does not occur, owing to the velocity discontinuity emerging at a singularity.

Solutions with limiting stresses in all zones

In the first instance the case where $\phi_{1,2,3,4}^{\mathrm{m}}=\phi$ will be considered. In general, solutions may be derived using the iterative approach described in Appendix 1. However, it is insightful to consider the range of distinct equilibrium stress fields that may be determined for all values of $\theta_{1}$ from 0 to $45+\phi / 2$. Values of $\theta_{1}, \theta_{2}, \theta_{3}, \xi_{1}, \xi_{2}$ are plotted against $\theta_{1}$ in Fig. 3 for the case $\phi=30^{\circ}$.

The above stress field solution may be used to determine the corresponding velocity characteristics that are orientated at $\pm \varepsilon_{\mathrm{V}}$ to the principal stress directions, where $\varepsilon_{\mathrm{v}}=$ $\pi / 4-\psi / 2$ for the coaxial flow rule.

For kinematic compatibility the line OE must be orientated at $\psi$ to the vertical away from the upward-displacing basement layer (or towards the downward-displacing basement layer), where $\psi$ is the angle of dilation. For this to occur the following relationship must exist between $\psi$ and $\xi$ in the yielding zone, as shown in Fig. 6.

$$
\xi+\varepsilon_{\mathrm{v}}=\theta_{\mathrm{E}}
$$

where $\theta_{\mathrm{E}}$ (angle $\left.\mathrm{A}_{0} \mathrm{OE}\right)$ is given by

$$
\theta_{\mathrm{E}}=\pi / 2-\psi
$$

Hence

$$
\psi=\pi / 2-2 \xi
$$

and

$$
\theta_{\mathrm{E}}=2 \xi
$$

However, there is only a narrow range of stress fields for which equation (4) is satisfied with $0<\psi<\phi$. If OE lies in zone $\mathrm{A}_{1} \mathrm{OA}_{2}$, then $\theta_{\mathrm{E}}$ must be less than $\theta_{2}$, and if OE lies in zone $\mathrm{A}_{2} \mathrm{OA}_{3}$, then $\theta_{\mathrm{E}}$ must be greater than $\theta_{2}$. Values of $\theta_{\mathrm{E}}$, determined from equation (5), for each zone are also plotted in Fig. 3, where $\theta_{\mathrm{E} 1}$ represents $\theta_{\mathrm{E}}$ derived for zone 1 $\left(\mathrm{A}_{1} \mathrm{OA}_{2}\right)$, and $\theta_{\mathrm{E} 2}$ represents $\theta_{\mathrm{E}}$ derived for zone $2\left(\mathrm{~A}_{2} \mathrm{OA}_{3}\right)$. For zone 1 , valid points are below point $A$ in Fig. 3 $\left(\theta_{\mathrm{E} 1}<\theta_{2}\right)$. These are impossible, as they imply $\psi>\phi$. However, in zone 2, valid values of $\theta_{\mathrm{E} 2}$ exist between points $\mathrm{B}$ and $\mathrm{C}$ in Fig. 3, giving a range of $0<\psi<14.48^{\circ}$.

For simple velocity fields of this nature, there exists a

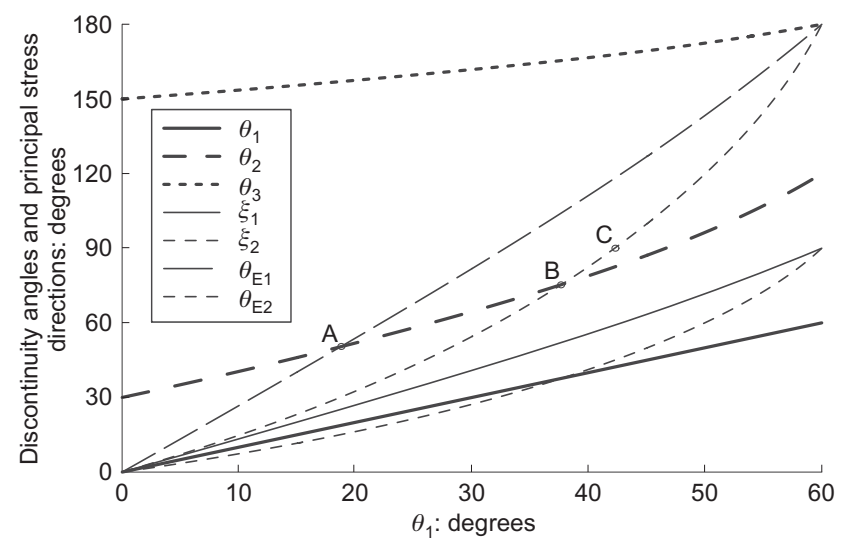

Fig. 3. Variation of discontinuity angles and major principal stress directions with $\theta_{1}$ for $\phi=30^{\circ}$ for a trapdoor underlying a weightless soil with surface load value $\psi_{\max }$ for each angle $\phi$ such that viable solutions occur only for $0<\psi<\psi_{\max }$. For $\psi>\psi_{\max }$ the simple velocity field solution is not possible. However, from a practical point of view, this is not a problem, as $\psi_{\max }$ typically exceeds that expected for real soils.

Velocity and stress characteristics are shown in Fig. 4 for an example problem with $\phi=30^{\circ}, \psi=4^{\circ}$ and $\theta_{1}=41^{\circ}$. The corresponding Mohr's circles are given in Fig. 5.

If the stress state (mean stress $\sigma$, major principal stress direction $\xi$ ) along $\mathrm{OE}$ is known, then the load on a finitewidth trapdoor or anchor may be determined from equation (1), where

$$
\Upsilon=\cot \theta_{\mathrm{E}}+\frac{\sigma}{\sin \theta_{\mathrm{E}}}\left[\sin \phi \cos \left(2 \xi-\theta_{\mathrm{E}}\right)-\cos \theta_{\mathrm{E}}\right]
$$

Values of $\Upsilon$ against $\phi$ are plotted for viable values of $\psi$ in $5^{\circ}$ increments in Fig. 7. Also plotted in this graph are the associative case upper and lower bounds $\Upsilon_{U}$ and $\Upsilon_{L}$. The upper bound is given by $\tan \phi$, as demonstrated later. The lower bound is found by the same method as described by Smith (1998) for soil possessing self-weight; all possible sets of $\theta_{1}, \theta_{2}, \theta_{3}$ are searched, and the value of $\theta_{\mathrm{E}}$ that gives the largest value of $\Upsilon$ determined. It is found that $\theta_{\mathrm{E}}=\theta_{2}$ always. It is seen that the lower and upper bounds are almost identical, thus defining the true associative load to within $4 \%$.

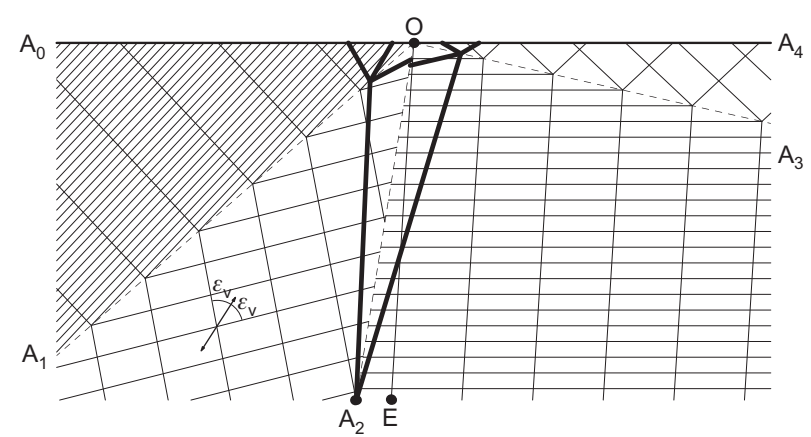

Fig. 4. Velocity characteristics (thin lines) for weightless soil with surface load $\left(\phi=30^{\circ}, \psi=4^{\circ}, \theta_{1}=41^{\circ}\right)$. Bold lines indicate the pattern of stress characteristics

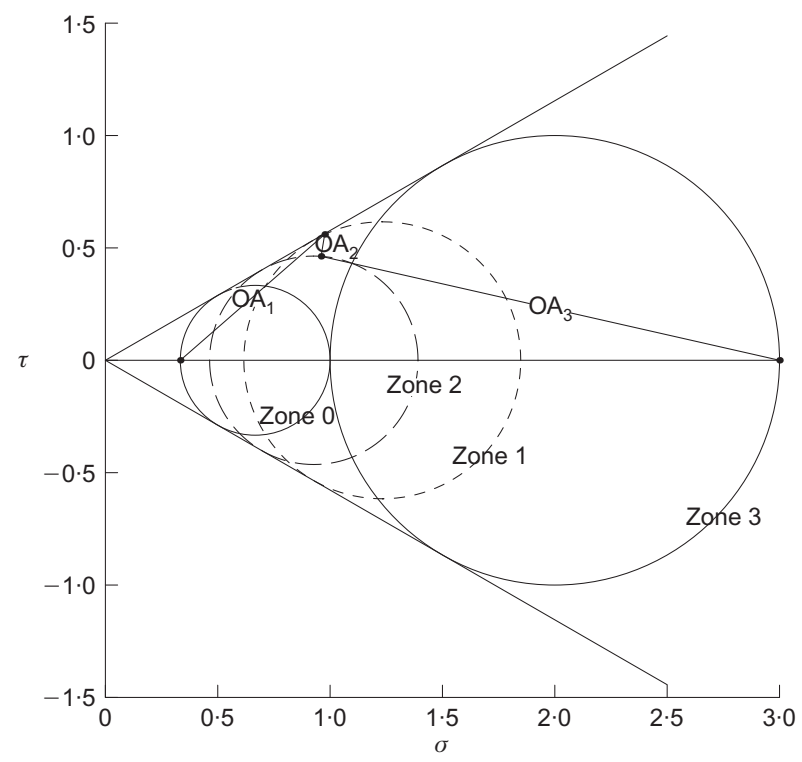

Fig. 5. Mohr's circles for the stress states in each zone for a weightless soil problem with surface load $q B=1\left(\phi=30^{\circ}, \psi=4^{\circ}\right.$, $\left.\theta_{1}=41^{\circ}\right)$ 


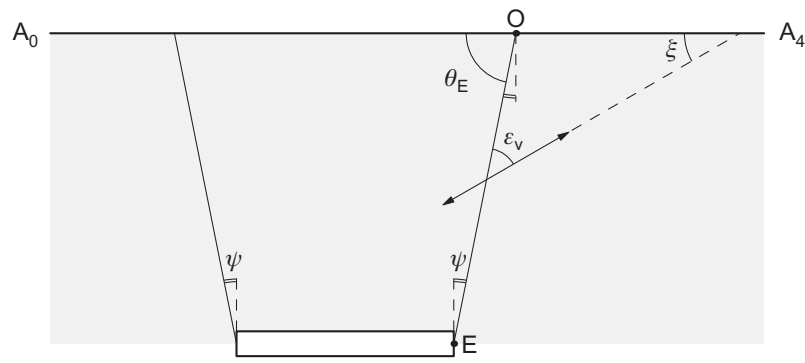

Fig. 6: Relationship between $\xi, \varepsilon_{\mathrm{v}}$, and $\psi$ and $\theta_{\mathrm{E}}$ on right-hand edge of sliding-wedge boundary

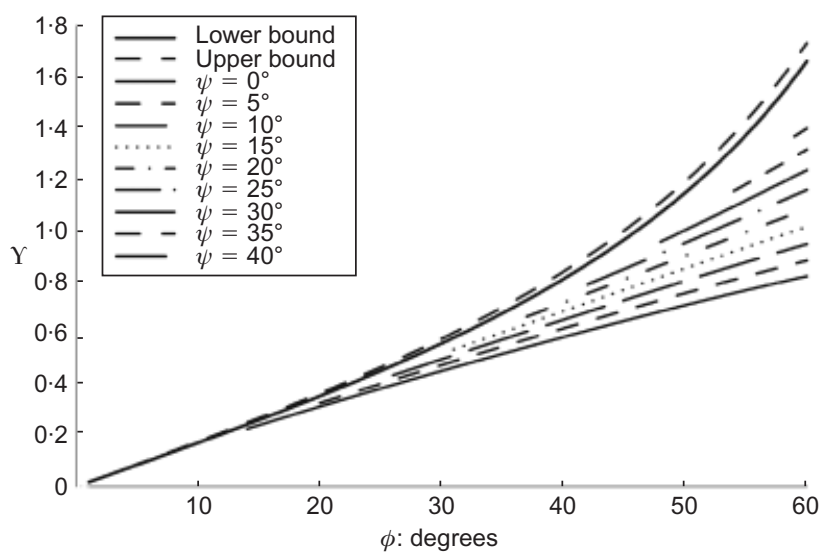

Fig. 7. Variation of associative upper and lower bounds and nonassociative values of $\Upsilon$ with $\phi$ for a trapdoor underlying a weightless soil with surface load

Solutions with yield only in deforming zone

So far, only solutions with yield in all zones have been considered. As stated earlier, yield is required only in the zone(s) where deformation takes place. Thus $\phi^{\mathrm{m}}$ may be lower than $\phi$ in all other zones. In these cases, it is found that the largest (smallest) anchor (trapdoor) capacity occurs with the assumption of $\phi^{\mathrm{m}}=\phi$ in all zones, and the lowest values occur for low values of $\phi_{3}^{\mathrm{m}}$. In all analyses carried out, it was found that $\Upsilon$ was unaffected by the value of $\phi_{0}^{\mathrm{m}}$ or $\phi_{1}^{\mathrm{m}}$ (except where an invalid solution was produced), and that the velocity discontinuity always lay in zone 2 . Selected results are presented in Table 1.

It is seen that values of $\Upsilon$ ranging from 0.333 to 0.451 are possible for $\phi=30^{\circ}$ and $\psi=0$ (compare with 0.577 for the associative case). The lowest value occurs for $\phi_{3}^{\mathrm{m}}=0$.

\section{SMITH}

This indicates that it may be possible to find a solution where the sense of the Mohr's circle in zone 3 is reversed, that is, where $\xi_{3}=90^{\circ}$. Solutions may in fact be found, and are listed in the second part of Table 1. In these cases it is possible to reach lower values of $\Upsilon$, down to 0.291 for $\phi=30^{\circ}$ and $\psi=0$, which is almost half that of the associative value.

Higher values are also possible if values of $\phi_{3}^{\mathrm{m}}>\phi$ are considered, as shown in the second part of Table 1. This may be a possibility where $\phi$ is considered a critical state value while $\phi_{3}^{\mathrm{m}}$ is considered a peak value.

Solutions for non-zero values of $\psi$ and for $\phi=40^{\circ}$ are given in the third and fourth parts of Table 1.

A wider range may exist if other solution types other than that considered in this paper exist.

\section{Scope of validity of solutions}

The solutions so derived may be considered to be valid for all depths (although not necessarily critical). For example, for an active trapdoor, $\mathrm{A}_{3}$ intersects the line of symmetry, as depicted in Fig. 8. However, a further discontinuity line may be employed downwards from $\mathrm{A}_{3}$ to the intersection with $\mathrm{OE}$ such that the major principal stress direction in the zone between it and the symmetry line remains horizontal. It is possible to show that this sequence of intersecting discontinuity zones can be continued downwards, but this is beyond the scope of this paper. Similar arguments may be made for the passive trapdoor case.

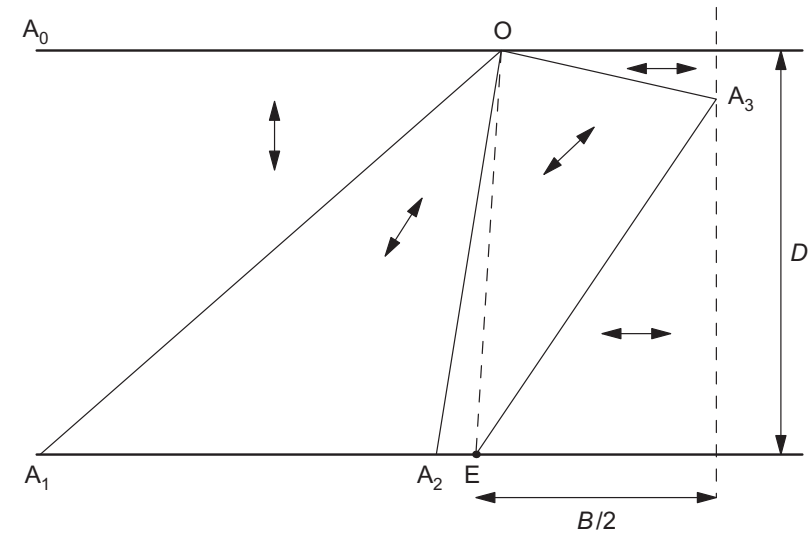

Fig. 8. Pattern of stress discontinuities required for determining the limit of validity of the active trapdoor problem. Trapdoor edge located at $\mathbf{E}$

Table 1. Example sets of equilibrium discontinuities for weightless soil showing range of possible $\Upsilon$ values for non-associative shearing

\begin{tabular}{|c|c|c|c|c|c|c|c|c|c|c|c|c|c|c|}
\hline$\phi$ & $\psi$ & $\varphi_{3}^{m}$ & $\sigma_{0}$ & $\xi_{0}$ & $\sigma_{1}$ & $\xi_{1}$ & $\sigma_{2}$ & $\xi_{2}$ & $\sigma_{3}$ & $\xi_{3}$ & $\theta_{1}$ & $\theta_{2}$ & $\theta_{3}$ & $\Upsilon$ \\
\hline $30^{\circ}$ & $0^{\circ}$ & $0^{\circ}$ & 0.67 & $90^{\circ}$ & 1.03 & $67 \cdot 5^{\circ}$ & 0.67 & $45 \cdot 0^{\circ}$ & $1 \cdot 0$ & $0^{\circ}$ & $47.5^{\circ}$ & $87.5^{\circ}$ & $135 \cdot 0^{\circ}$ & 0.333 \\
\hline $30^{\circ}$ & $0^{\circ}$ & $10^{\circ}$ & 0.67 & $90^{\circ}$ & $1 \cdot 11$ & $63 \cdot 5^{\circ}$ & 0.77 & $45 \cdot 0^{\circ}$ & $1 \cdot 21$ & $0^{\circ}$ & $45.0^{\circ}$ & $85 \cdot 1^{\circ}$ & $149.3^{\circ}$ & 0.386 \\
\hline $30^{\circ}$ & $0^{\circ}$ & $20^{\circ}$ & 0.67 & $90^{\circ}$ & $1 \cdot 16$ & $60 \cdot 8^{\circ}$ & $0 \cdot 85$ & $45 \cdot 0^{\circ}$ & 1.52 & $0^{\circ}$ & $43 \cdot 4^{\circ}$ & $83 \cdot 5^{\circ}$ & $160 \cdot 4^{\circ}$ & 0.424 \\
\hline $30^{\circ}$ & $0^{\circ}$ & $30^{\circ}$ & 0.67 & $90^{\circ}$ & 1.20 & $59 \cdot 1^{\circ}$ & 0.90 & $45 \cdot 0^{\circ}$ & $2 \cdot 0$ & $0^{\circ}$ & $42 \cdot 3^{\circ}$ & $82.6^{\circ}$ & $167.9^{\circ}$ & 0.451 \\
\hline $30^{\circ}$ & $0^{\circ}$ & $7^{\circ}$ & 0.67 & $90^{\circ}$ & 0.96 & $71 \cdot 3^{\circ}$ & 0.58 & $45 \cdot 0^{\circ}$ & 0.89 & $90^{\circ}$ & $49 \cdot 8^{\circ}$ & $89.8^{\circ}$ & $124 \cdot 8^{\circ}$ & 0.291 \\
\hline $30^{\circ}$ & $0^{\circ}$ & $40^{\circ}$ & 0.67 & $90^{\circ}$ & $1 \cdot 22$ & $58.0^{\circ}$ & 0.94 & $45 \cdot 0^{\circ}$ & $2 \cdot 8$ & $0^{\circ}$ & $41 \cdot 6^{\circ}$ & $81.9^{\circ}$ & $172 \cdot 7^{\circ}$ & 0.470 \\
\hline $30^{\circ}$ & $7^{\circ}$ & $30^{\circ}$ & 0.67 & $90^{\circ}$ & $1 \cdot 26$ & $55.9^{\circ}$ & 0.95 & $41.5^{\circ}$ & $2 \cdot 0$ & $0^{\circ}$ & $40 \cdot 2^{\circ}$ & $79 \cdot 2^{\circ}$ & $166 \cdot 7^{\circ}$ & 0.484 \\
\hline $30^{\circ}$ & $7^{\circ}$ & $6.9^{\circ}$ & 0.67 & $90^{\circ}$ & $1 \cdot 13$ & $62 \cdot 4^{\circ}$ & 0.75 & $41.5^{\circ}$ & $1 \cdot 14$ & $0^{\circ}$ & $44 \cdot 3^{\circ}$ & $83.0^{\circ}$ & $141.9^{\circ}$ & 0.410 \\
\hline $30^{\circ}$ & $14^{\circ}$ & $30^{\circ}$ & 0.67 & $90^{\circ}$ & 1.32 & $52.5^{\circ}$ & 1.00 & $38.0^{\circ}$ & $2 \cdot 0$ & $0^{\circ}$ & $37.9^{\circ}$ & $75 \cdot 8^{\circ}$ & $165 \cdot 6^{\circ}$ & 0.514 \\
\hline $40^{\circ}$ & $0^{\circ}$ & $40^{\circ}$ & 0.61 & $90^{\circ}$ & 1.39 & $59 \cdot 7^{\circ}$ & 0.91 & $45 \cdot 0^{\circ}$ & $2 \cdot 8$ & $0^{\circ}$ & $46 \cdot 7^{\circ}$ & $78 \cdot 1^{\circ}$ & $171.0^{\circ}$ & 0.583 \\
\hline $40^{\circ}$ & $0^{\circ}$ & $0^{\circ}$ & 0.61 & $90^{\circ}$ & $1 \cdot 14$ & $67 \cdot 5^{\circ}$ & $0 \cdot 61$ & $45 \cdot 0^{\circ}$ & $1 \cdot 0$ & $0^{\circ}$ & $52 \cdot 0^{\circ}$ & $83.0^{\circ}$ & $135.0^{\circ}$ & 0.391 \\
\hline $40^{\circ}$ & $12^{\circ}$ & $40^{\circ}$ & $0 \cdot 61$ & $90^{\circ}$ & 1.57 & $54 \cdot 1^{\circ}$ & 1.02 & $39.0^{\circ}$ & $2 \cdot 80$ & $0^{\circ}$ & $42 \cdot 8^{\circ}$ & $72 \cdot 4^{\circ}$ & $169 \cdot 5^{\circ}$ & 0.665 \\
\hline $40^{\circ}$ & $12^{\circ}$ & $3 \cdot 8^{\circ}$ & $0 \cdot 61$ & $90^{\circ}$ & $1 \cdot 28$ & $63 \cdot 0^{\circ}$ & $0 \cdot 66$ & $39.0^{\circ}$ & 1.07 & $0^{\circ}$ & $48.9^{\circ}$ & $78 \cdot 0^{\circ}$ & $133.8^{\circ}$ & 0.504 \\
\hline $40^{\circ}$ & $23^{\circ}$ & $40^{\circ}$ & 0.61 & $90^{\circ}$ & $1 \cdot 76$ & $48.5^{\circ}$ & $1 \cdot 14$ & $33.5^{\circ}$ & $2 \cdot 80$ & $0^{\circ}$ & $38.6^{\circ}$ & $66 \cdot 8^{\circ}$ & $168.0^{\circ}$ & 0.737 \\
\hline
\end{tabular}


SOIL POSSESSING SELF-WEIGHT WITH NO SURFACE LOAD

The method of generating limiting stress fields for such problems has been described in detail by Smith (1998), who used the approach to generate lower-bound solutions to the associative flow case. Where the soil is assumed to have uniformly distributed properties of angle of friction $(\phi)$, angle of dilation $(\psi)$ and self-weight $\gamma$, then the limit load $F$ per unit length for the anchor or trapdoor may be expressed in a dimensionless form as

$$
\frac{F}{\gamma D B}=1 \pm \frac{D}{B} \Psi(\phi, \psi)
$$

where the sign depends on whether displacement is active $(-)$ or passive $(+)$.

While a similar pattern of three stress discontinuities is required to generate valid solutions, the stress characteristics in zones $\mathrm{A}_{1} \mathrm{OA}_{2}$ and $\mathrm{A}_{2} \mathrm{OA}_{3}$ are curved, owing to the soil self-weight. However, velocity characteristics may still be generated from the stress fields, as outlined for the weightless soil, and simple velocity fields with a single velocity discontinuity can be found, as depicted in Fig. 9. From these

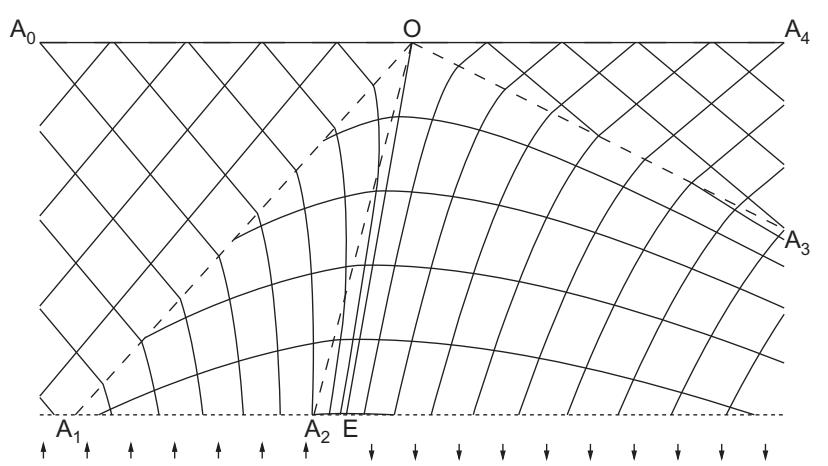

Fig. 9. Velocity characteristics for soil possessing self-weight with no surface load $\left(\phi=30^{\circ}, \psi=10^{\circ}\right)$. Trapdoor edge located at $\mathrm{E}$ solutions, values of $\Psi$ and $\psi_{\max }$ may be found for each value of $\phi$. In this paper solutions are presented only for the assumption of limiting conditions in all zones. Values of $\Psi$ are given in Fig. 10, together with values of the associative upper and lower bounds $\Psi_{\mathrm{U}}$ and $\Psi_{\mathrm{L}}$. The methodology for determining solution validity limits on the embedment ratio $D / B$ for the case of soil possessing self-weight has been outlined by Smith (1998).

\section{INTERPRETATION OF THE LIMIT LOAD}

Unlike associative solutions, non-associative solutions are not necessarily unique, and a number of solutions may exist, depending on initial conditions and the stress path to failure. The work here attempts to bracket the range of possible solutions. For example, for the solution type depicted in Fig. 4 it is not necessary that the soil in zones 1, 2, 3 and 4 be in a state of limiting equilibrium, as the only plastic deformation is taking place in zone 2. If non-limiting states are considered in these zones, then values of $\Upsilon$ and $\Psi$ lower than for the full limiting case are obtained (as would be expected), and $\psi_{\max }$ also reduces.

It is suggested that higher non-associative limit loads are unlikely to be found. The solutions presented are likely to be an upper bound to the set of non-associative solutions.

\section{Comparison with finite-element and experimental data}

Rowe \& Davis (1982) undertook elasto-plastic FE analysis of the anchor uplift problem, and presented data for both $\psi=0$ and $\psi=\phi$ cases. These are plotted in Fig. 10 (averaged from data for $D / B=1,2$ and 3) and show excellent agreement, providing support for the current theory. Examination of the velocity fields presented by Rowe \& Davis (1982) for shallow anchors also shows similar behaviour to that predicted by the current theory.

White et al. (2008) provided an extensive survey of the experimental data available in the literature for model anchor and pipe tests, together with a proposed limit equilibrium

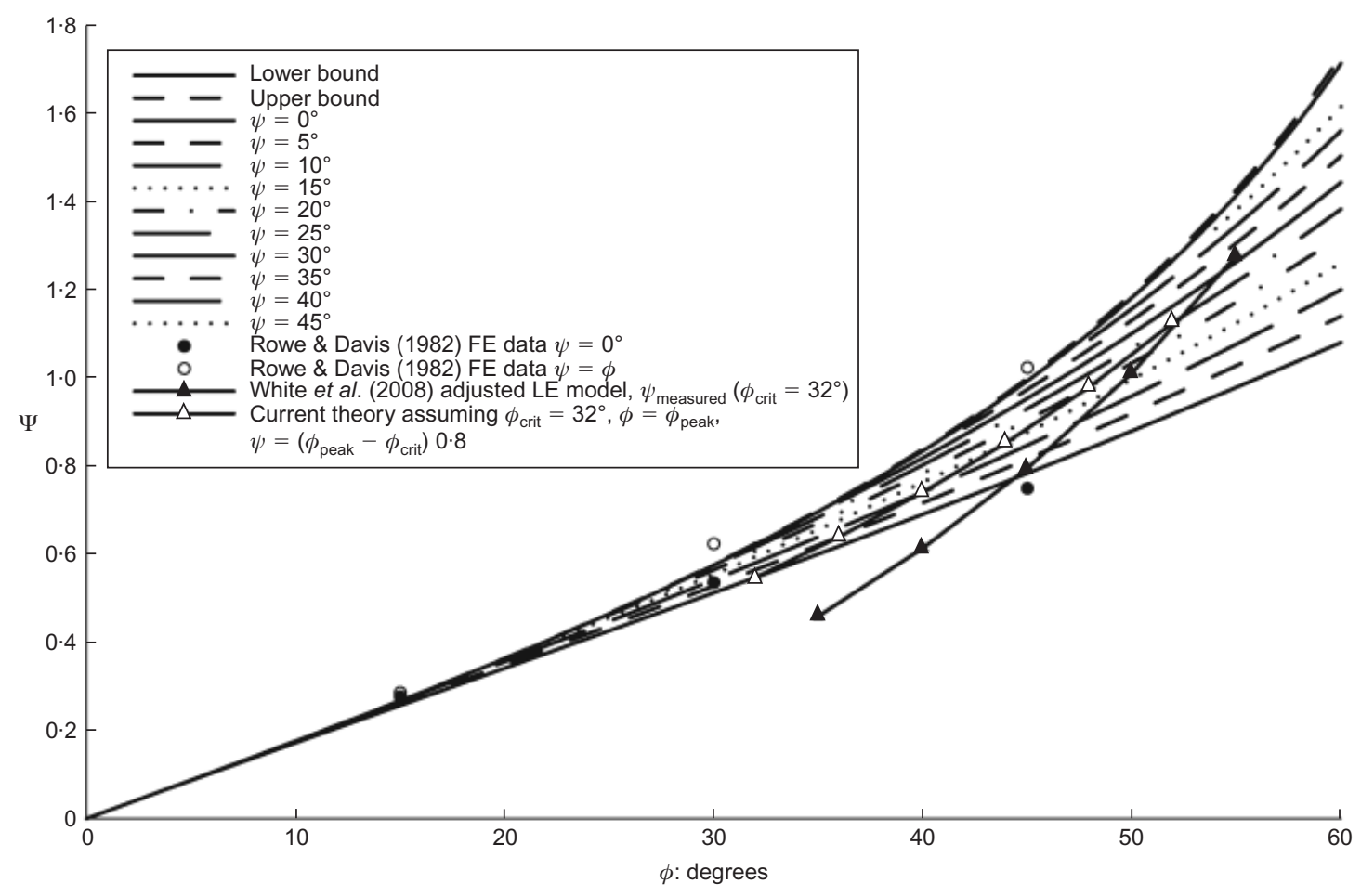

Fig. 10. Variation of associative upper and lower bounds and non-associative values of $\Psi$ with $\phi$ for a trapdoor underlying a soil possessing self-weight with no surface load 
model for predicting anchor and pipe uplift capacity that requires values of $\phi_{\text {crit }}, \phi_{\text {peak }}$ and $\psi$ (see Appendix 2). They demonstrated that their model underpredicts the anchor data on average by $14 \%$, as shown in Fig. 11 , in which their anchor data are reproduced (where $N_{\gamma}=F / \gamma D B$ ). This underprediction is averaged over all burial depth/width ratios, and unfortunately (a) does not directly include dilation data, and (b) is also in terms of $N_{\gamma}=1+\Psi(D / B)$ rather than $\Psi$, which is where the uncertainty lies. However, an approximate comparison can be made. Examination of Fig. 11, where $N_{\gamma}$ is plotted against burial depth ratio, indicates broad increase of error with burial depth ratio to the order of $28 \%$ (approximately double the average, as expected) at $D / B=8$, that is, at a gradient of $0 \cdot 28 / 8=0 \cdot 035$. Thus

$$
N_{\gamma, \text { measured }} \approx \frac{N_{\gamma, \text { predicted }}}{1-0.035 D / B}=\frac{1+(D / B) \Psi_{\mathrm{W}}}{1-0.035(D / B)}
$$

where $\Psi_{\mathrm{W}}$ is the predicted value of $\Psi$ from White et al. (2008); see Appendix 2. Hence

$$
\begin{aligned}
N_{\gamma, \text { measured }} & \approx\left(1+\frac{D}{B} \Psi_{\mathrm{W}}\right)\left(1+0.035 \frac{D}{B}\right) \\
& \approx 1+\left[\Psi_{\mathrm{W}}\left(1+0.035 \frac{D}{B}\right)+0.035\right] \frac{D}{B}
\end{aligned}
$$

To avoid the $(D / B)^{2}$ term, the middle term on the right-hand side will be approximately taken at $D / B=4$. Hence

$$
\Psi_{\text {measured }} \approx 1 \cdot 14 \Psi_{\mathrm{W}}+0.035
$$

The values of $\Psi_{\text {measured }}$ assuming $\phi_{\text {crit }}=32^{\circ}, \phi=\phi_{\text {peak }}$ and $\psi=\left(\phi_{\text {peak }}-\phi_{\text {crit }}\right) / 0 \cdot 8$, as proposed in White et al. (2008), are plotted in Fig. 10 for values of $\phi=35-55^{\circ}$, together with values predicted by the current theory for the same parameters. It is seen that the modified White et al. (2008) values are reasonable fits to the current data at the higher values of $\phi$. The lower values are still within the expected range of the current theory, based on the trends observed for the weightless soil problem. Of course, the current model pertains to shallow anchors only, and the comparison is seen through the filter of the White et al. (2008) limit equilibrium solution. Also, other effects such as geometrical change prior to failure have not been accounted for.

Broadly, the FE and experimental data seem to support the assumption that yield is occurring in all zones. On this basis, based on Fig. 10, non-associativity leads to an approximate $12 \%$ reduction in the value of $\Psi$, compared with the associative value for practical cases.

\section{COMBINED ANALYSIS FOR SOIL POSSESSING SELF-}

\section{WEIGHT AND SUBJECTED TO SURFACE LOAD}

Translational failure mechanism analysis of combined problem

A simple translational mechanism appropriate to the trapdoor/anchor problem has been outlined by Murray \& Geddes (1987). This employs two velocity discontinuities emerging upwards from each trapdoor edge, as illustrated for the passive case in Fig. 12.

The analysis presented here extends previous analyses by considering both self-weight and surface loading. From the corresponding force polygon relating $U, V$ and $W$ it can be shown that

$$
V=\frac{W \cos (\alpha-\phi)}{\sin (\alpha+\beta)}
$$

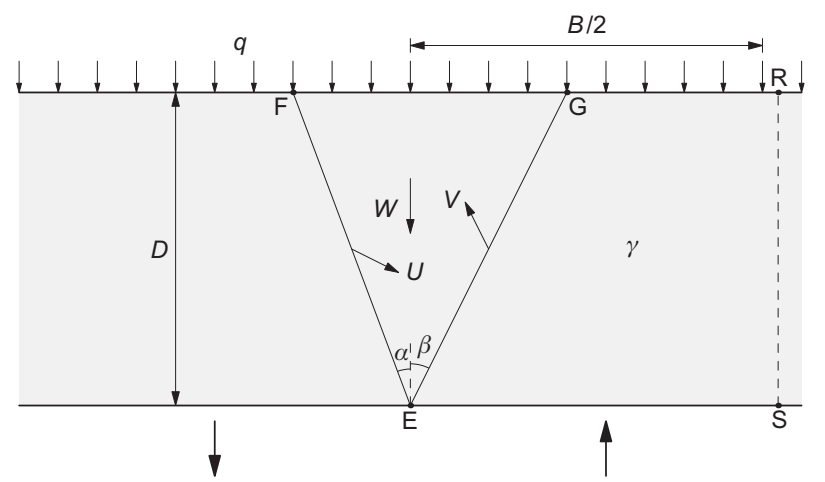

Fig. 12. Translational failure mechanism for passive trapdoor showing interface force resultants $U$ and $V$ and block EFG selfweight $W$

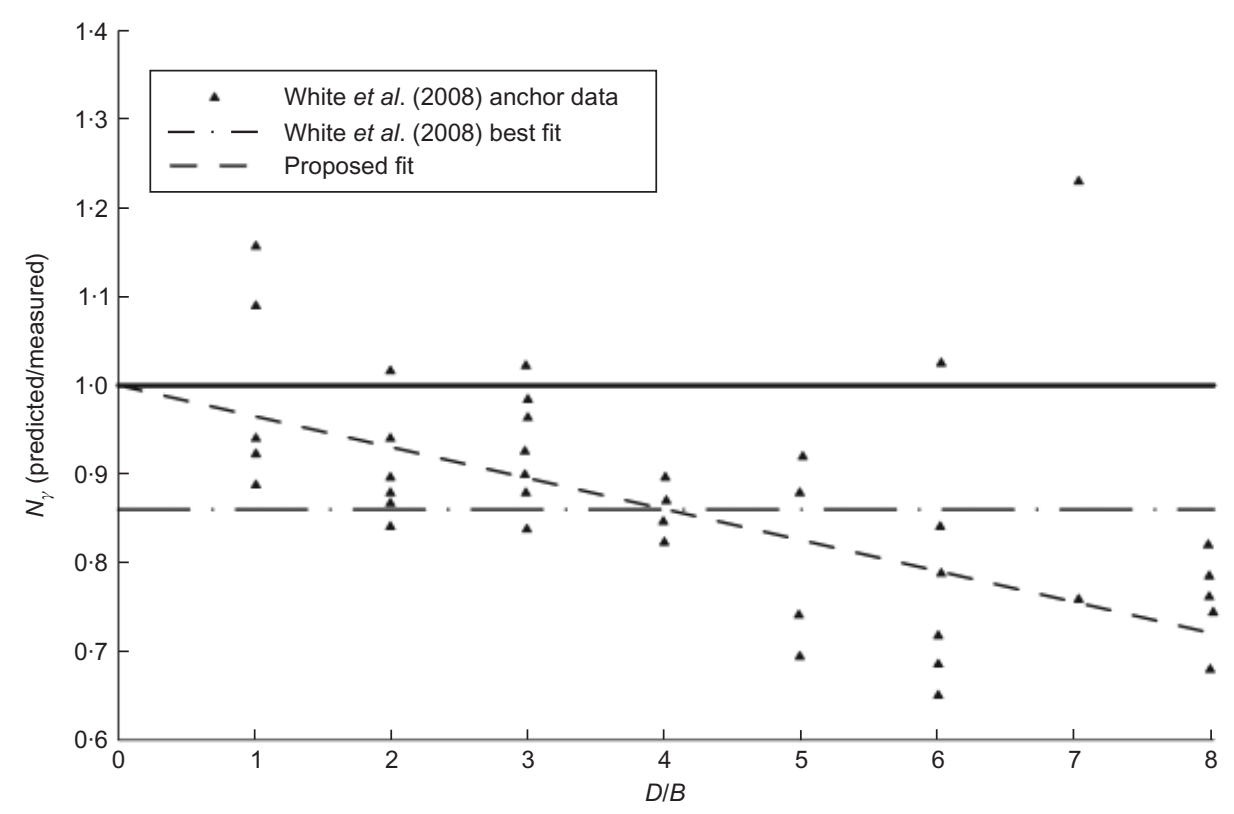

Fig. 11. Anchor data reproduced from White et al. (2008) Fig. 5(d), illustrating the best fit proposed by White et al. (2008) and alternative fit proposed in the current work 
where $W$ is given by the self-weight and surface loading on triangle EFG

$$
W=\left(q D+\frac{1}{2} \gamma D^{2}\right)(\tan \alpha+\tan \beta)
$$

The actual force per unit length, $F_{\mathrm{p}}$, on the (passive) half trapdoor ES is given by

$$
\begin{aligned}
& \frac{F_{\mathrm{p}}}{B}=q\left(1-\frac{2 D}{B} \tan \beta\right)+\gamma D\left(1-\frac{D}{B} \tan \beta\right)+\frac{2 V}{B} \sin (\phi+\beta) \\
& \frac{F_{\mathrm{p}}}{B}=q\left(1+\frac{2 D}{B} Y_{\mathrm{p}}\right) \gamma D\left(1+\frac{D}{B} Y_{\mathrm{p}}\right)
\end{aligned}
$$

where

$$
Y_{\mathrm{p}}=-\tan \beta+\frac{\sin (\phi+\beta) \cos (\alpha-\phi)(\tan \alpha+\tan \beta)}{\sin (\alpha+\beta)}
$$

the subscript $\mathrm{p}$ denoting the passive case. In general, for both active (a) and passive (p) cases, it can be shown that

$$
\frac{1}{B}\left\{\begin{array}{l}
F_{\mathrm{p}} \\
F_{\mathrm{a}}
\end{array}\right\}=q\left(1 \pm \frac{2 D}{B} Y\right)+\gamma D\left(1 \pm \frac{D}{B} Y\right)
$$

and

$$
\left.\begin{array}{l}
Y_{\mathrm{p}} \\
Y_{\mathrm{a}}
\end{array}\right\}=-\tan ( \pm \beta)+\frac{\sin (\phi \pm \beta) \cos (\alpha \mp \phi)(\tan \alpha+\tan \beta)}{\sin (\alpha+\beta)}
$$

For an upper bound associative analysis, $Y$ must be minimised, subject to the kinematic constraints $\alpha \geqslant \phi$ for the passive case or $\beta \geqslant \phi$ for the active case. This occurs when either of the velocity discontinuities is orientated at angle $\phi$ to the vertical away from the part of the basement layer that is undergoing upward relative movement. In either case equation (17) reduces to

$$
\Upsilon_{\mathrm{U}}=\Psi_{\mathrm{U}}=Y_{\min }=\tan \phi
$$

At this stage, kinematically, the other discontinuity vanishes for the associative case. The solution is valid for all depth ratios $D / B$ for the passive case, and for $D / B<1 /(2 \tan \phi)$ for the active case. For the active case for $D / B>1 /(2 \tan \phi)$ a kinematically admissible solution is one involving a single displacing isosceles triangle of soil whose base is defined by the trapdoor and whose apex angle equals $2 \phi$. No deformation reaches the surface, and yield parameters $\Upsilon_{\mathrm{Ua}}=0$ and $\Psi_{\mathrm{Ua}}=B[1-B /(4 D \tan \phi)] / D$.

For the non-associative case, values of $Y$ may be larger or smaller than as given in equation (18), as the kinematic constraints are less restrictive on $\alpha$ and $\beta$, and no obvious distinct solution presents itself.

\section{General limit loads for soil possessing self-weight and subjected to surface load}

In common with the classic Terzaghi bearing capacity equation for footings, it is proposed that the load for an anchor/trapdoor underlying a soil possessing self-weight with surface self-weight may be approximated by equation (19) as

$$
p=q N_{q}+\gamma D N_{\gamma}
$$

where

$$
\begin{aligned}
& N_{q}=1 \pm \frac{2 D}{B} \Upsilon \\
& N_{\gamma}=1 \pm \frac{D}{B} \Psi
\end{aligned}
$$

where the + sign corresponds to the passive case and the sign to the active case.

For an associative soil, the upper bounds combine exactly as indicated in equation (19) (as derived in equation (16)). Applying the principles of superposition to the lower-bound case, as detailed by Bolton \& Lau (1993) for the bearing capacity problem, it is easily shown that superposition of the lower-bound limiting stress fields will always lead to a equilibrium stress field that nowhere violates yield (but is not necessarily everywhere in yield). Thus the combined lower-bound solutions will also be a lower bound. As the individual lower bounds are almost identical to their respective upper bounds, then the combined lower bound will be almost identical to the combined upper bound. Thus equation (19) is closely applicable to the associative case.

For the non-associative case, the above arguments are not applicable, but it is not unreasonable to propose that the above equation will give a good approximation to the combined load, particularly for large values of dilatancy.

\section{CONCLUSIONS}

(a) A class of complete equilibrium limiting stress fields and compatible velocity fields for the statically indeterminate shallow anchor/trapdoor problem in non-associative cohesionless soils has been presented. The analysis utilises the coaxial flow rule, and can be used to predict the range of limit loads on both active and passive trapdoors (anchors). For $\phi=30^{\circ}, \psi=0$, for example, possible limit loads down to approximately $50 \%$ of the associative limit load could be found for the problem scenario with surface surcharge and weightless soil.

(b) Very good agreement is demonstrated with FE data in the literature, and generally good agreement with experimental data, which is indicative that an analysis assuming general yield in the vicinity of an anchor edge is appropriate. For anchors overlain by typical real soils this would imply that the component of normalised nonassociative limit load $(F / \gamma D B)$ that varies with depth/ width ratio $(D / B)$ is approximately $12 \%$ lower than the corresponding associative limit load.

(c) The solutions presented in this paper illustrate the general principle that statically indeterminate problems can have non-associative limit loads that differ from the associative limit loads, owing to the indeterminacy.

(d) The analysis covers both cases of weightless soil with surface loading and cases of soil possessing self-weight with no surface loading and presents an equation that may be used to predict the combined case. This combined equation is shown to provide solutions for the associative case to within a few per cent of the true values.

\section{NOTATION}

$B$ breadth of anchor or trapdoor

$D$ depth of anchor or trapdoor

$F$ force per unit length on anchor or trapdoor

$N_{q}, N_{\gamma}$ dimensionless load factors

$p$ average limit vertical stress on anchor or trapdoor

$q$ soil surface surcharge stress

$r, \theta$ polar coordinates

$Y$ dimensionless load function for translational mechanism analysis

$\gamma \quad$ unit weight of soil

$\delta$ roughness of anchor or trapdoor 
$\varepsilon$ angle of stress characteristics to major principal stress direction

$\varepsilon_{\mathrm{V}}$ angle of velocity characteristics to major principal stress direction

$\xi$ orientation of principal stress direction to horizontal

$\sigma$ mean stress

$\sigma_{\mathrm{n}}, \tau$ normal and shear stresses

$\Upsilon$ dimensionless load function for weightless soil problem

$\phi$ angle of shearing resistance

$\phi^{\mathrm{m}}$ mobilised angle of shearing resistance

$\phi_{\text {crit }}$ critical state angle of shearing resistance

$\phi_{\text {peak }}$ peak angle of shearing resistance

$\Psi$ dimensionless load function for soil possessing self-weight problem

$\psi$ dilatancy angle

\section{APPENDIX 1: ANALYSIS OF TRAPDOOR UNDERLYING WEIGHTLESS SOIL WITH A SURFACE LOAD AND \\ VARIABLE MOBILISED STRENGTHS}

Consider the change in stress state passing from zone $i$ to zone $i+1$.

Let discontinuity between zone $i$ and zone $i+1$ be orientated at angle $\theta_{i+1}$. Let $S_{i}$ denote the centre of the Mohr's circle (with stress magnitude $\sigma_{i}$ ) representing the state of stress in zone $i$ and $I_{i+1}$ represent the intersection of the two Mohr's circles representing the state of stress in zones $i$ and $i+1$. As indicated in Fig. 13, it can be shown that

$$
\begin{aligned}
S_{i+1} \hat{S}_{i} I_{i+1} & =\pi+2\left(\theta_{i+1}-\xi_{i}\right) \\
S_{i} \hat{S}_{i+1} I_{i+1} & =2\left(\xi_{i+1}-\theta_{i+1}\right) \\
S_{i+1} \hat{I}_{i+1} S_{i} & =\pi-\left[\pi+2\left(\theta_{i+1}-\xi_{i}\right)\right]-2\left(\xi_{i+1}-\theta_{i+1}\right) \\
& =2\left(\xi_{i}-\xi_{i+1}\right)
\end{aligned}
$$

Applying the cosine rule to triangle $S_{i} I_{i+1} S_{i+1}$ gives

$$
\begin{aligned}
\left(\sigma_{i}-\sigma_{i+1}\right)^{2}= & \sigma_{i}^{2} \sin ^{2} \phi_{i}^{\mathrm{m}}+\sigma_{i+1}^{2} \sin ^{2} \phi_{i+1}^{\mathrm{m}} \\
& -2 \sigma_{i} \sin \phi_{i}^{\mathrm{m}} \sigma_{i+1} \sin \phi_{i+1}^{\mathrm{m}} \cos 2\left(\xi_{i}-\xi_{i+1}\right)
\end{aligned}
$$

or

$$
\begin{aligned}
\sigma_{i}^{2}+\sigma_{i+1}^{2}-2 \sigma_{i} \sigma_{i+1}= & \sigma_{i}^{2} \sin ^{2} \phi_{i}^{\mathrm{m}}+\sigma_{i+1}^{2} \sin ^{2} \phi_{i+1}^{\mathrm{m}} \\
& -2 \sigma_{i} \sin \phi_{i}^{\mathrm{m}} \sigma_{i+1} \sin \phi_{i+1}^{\mathrm{m}} \cos 2\left(\xi_{i}-\xi_{i+1}\right)
\end{aligned}
$$

If $\sigma_{i}$ is known, then $\sigma_{i+1}$ can be found from the solution of the quadratic equation

$$
\begin{aligned}
\sigma_{i+1}^{2}\left(1-\sin ^{2} \phi_{i+1}^{\mathrm{m}}\right) & -\sigma_{i+1}\left\{2 \sigma _ { i } \left[1-2 \sin \phi_{i}^{\mathrm{m}} \sin \phi_{i+1}^{\mathrm{m}}\right.\right. \\
& \left.\left.\times \cos 2\left(\xi_{i}-\xi_{i+1}\right)\right]\right\} \\
& +\sigma_{i}^{2}\left(1-\sin ^{2} \phi_{i}^{\mathrm{m}}\right)=0
\end{aligned}
$$

There are two roots to this quadratic equation, leading to two valid solutions. Solutions may be derived as follows.

$$
\begin{aligned}
& \sigma_{0}=\frac{q}{1+\sin \phi_{0}^{\mathrm{m}}} \\
& \xi_{0}=\pi / 2 \\
& \xi_{3}=0
\end{aligned}
$$

Assuming the velocity discontinuity is in zone 2,

$$
\begin{aligned}
& \xi_{2}+\varepsilon_{\mathrm{v}}+\psi=\pi / 2 \\
& \varepsilon_{\mathrm{v}}=\pi / 4-\psi / 2 \\
& \xi_{2}=\pi / 4-\psi / 2
\end{aligned}
$$

and

$$
\phi_{2}^{\mathrm{m}}=\phi
$$

If $\xi_{1}$ is known, then the values of $\sigma_{1}, \sigma_{2}$ and $\sigma_{3}$ can be computed using equation (27). A solution is thus found by finding the value of $\xi_{1}$ that gives

$$
\sigma_{3}=\frac{q}{1-\sin \phi_{3}^{\mathrm{m}}}
$$

The value of $\xi_{1}$ must typically found by iteration. Typically it is found that it is necessary to take the roots of equation (27) with the positive square root terms for discontinuities 1 and 3 and the negative for discontinuity 2 .

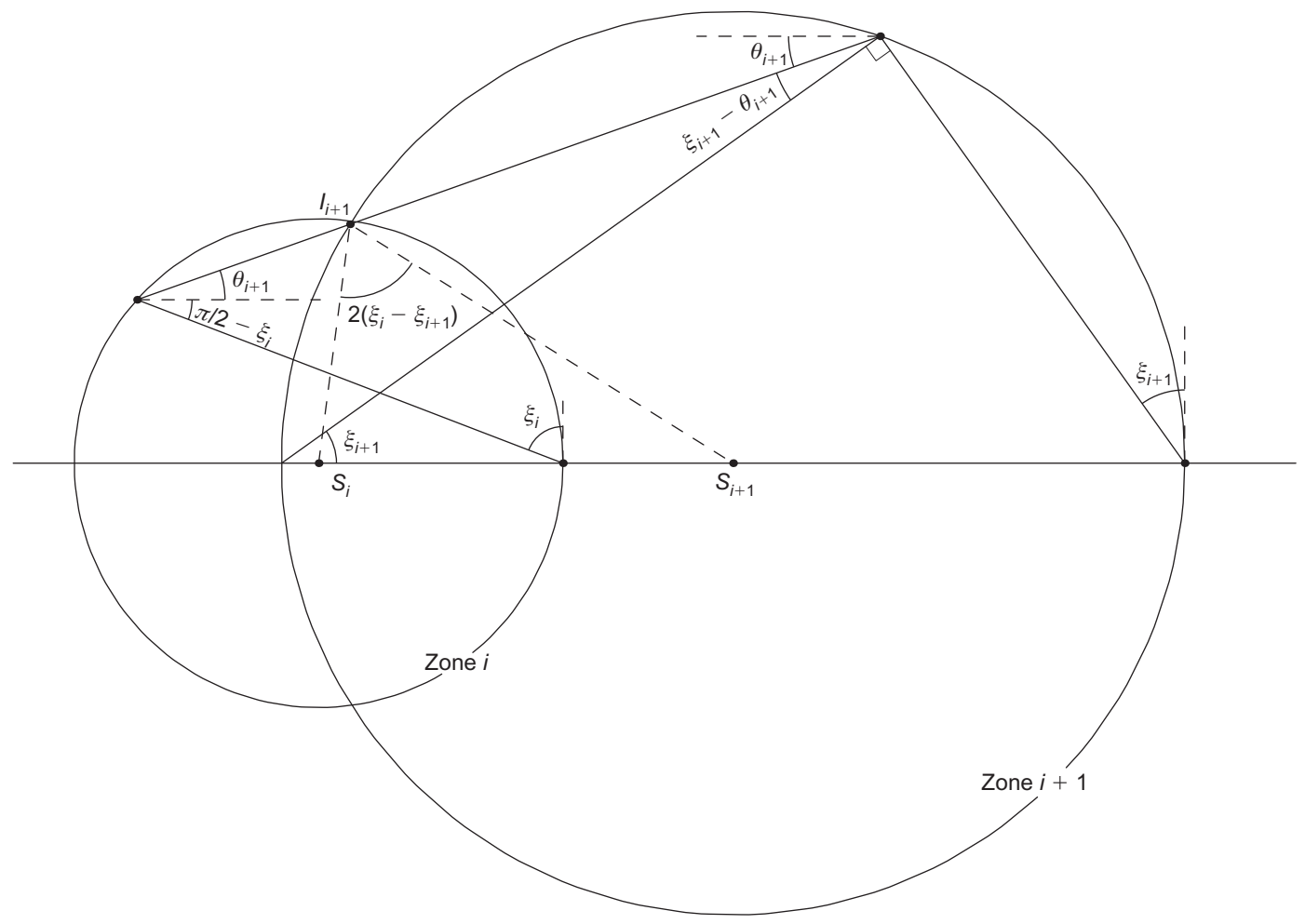

Fig. 13. Stress states at intersection of Mohr's circles 


\section{APPENDIX 2: WHITE ET AL. (2008) LIMIT}

EQUILIBRIUM ANALYSIS

The core limit equilibrium equations presented by White et al. (2008) are reproduced below (in current notation).

$$
\begin{aligned}
& \frac{F}{\gamma D B}=1+\frac{D}{B} \Psi\left(\phi_{\text {peak }}, \phi_{\text {crit }}, \psi\right) \\
& \Psi=\tan \psi+\left(\tan \phi_{\text {peak }}-\tan \psi\right)\left[\frac{1+K_{0}}{2}-\frac{\left(1-K_{0}\right) \cos 2 \psi}{2}\right] \\
& K_{0}=1-\sin \phi_{\text {crit }}
\end{aligned}
$$

\section{REFERENCES}

Bolton, M. \& Lau, C. (1993). Vertical bearing capacity factors for circular and strip footings on Mohr-Coulomb soil. Can. Geotech. J. 30, No. 6, 1024-1033.

Chen, W. F. (1975). Limit analysis and soil plasticity. Developments in Geotechnical Engineering 7. Amsterdam: Elsevier.
Davis, E. (1968). Theories of plasticity and the failure of soil masses. In Soil mechanics: Selected topics (ed. I. K. Lee), pp. 341-380. London: Butterworth.

Drescher, A. \& Detournay, E. (1993). Limit load in translational failure mechanisms for associative and non-associative materials. Géotechnique 43, No. 3, 443-456, http://dx.doi.org/10.1680/ geot.1993.43.3.443.

Murray, E. \& Geddes, J. (1987). Uplift of anchor plates in sand. $J$ Geotech. Engng Div. ASCE 113, No. 3, 202-215.

Rowe, R. \& Davis, E. (1982). The behaviour of anchor plates in sand. Géotechnique 32, No. 1, 25-41, http://dx.doi.org/10.1680/ geot.1982.32.1.25.

Smith, C. (1998). Limit loads for an anchor/trapdoor embedded in an associative Coulomb soil. Int. J. Numer. Anal. Meth. Geomech. 22, No. 11, 855-865.

Vermeer, P. (1990). The orientation of shear bands in biaxial tests. Géotechnique 40, No. 2, 223-236, http://dx.doi.org/10.1680/ geot.1990.40.2.223.

White, D., Cheuk, C. \& Bolton, M. (2008). The uplift resistance of pipes and plate anchors buried in sand. Géotechnique 58, No. 10, 771-779, http://dx.doi.org/10.1680/geot.2008.3692. 